\title{
Effects of various surgical protocols on maxillofacial growth in patients with unilateral cleft lip and palate: a systematic review
}

\author{
Pasquier Corthouts ${ }^{1, \#}$, Fien Boels ${ }^{1, \#}$, Elke Van de Casteele ${ }^{1,2,3}$, Nasser Nadjmi $^{1,2,3,4}$ \\ ${ }^{1}$ Faculty of Medicine \& Health Sciences, University of Antwerp, Campus Drie Eiken, Antwerp 2610, Belgium. \\ ${ }^{2}$ Department of Cranio-Maxillofacial Surgery, Antwerp University Hospital, Edegem 2650, Belgium. \\ ${ }^{3}$ All for Research VZW, Antwerp 2018, Belgium. \\ ${ }^{4}$ Department of Cranio-Maxillofacial Surgery, ZMACK, AZ MONICA Antwerp, Antwerp 2018, Belgium. \\ "Co first authors.
}

Correspondence to: Prof. Nasser Nadjmi, Department of Cranio-Maxillofacial Surgery, University of Antwerp, AZ MONICA Antwerp, Harmoniestraat 68, Antwerp 2018, Belgium. E-mail: nasser@nadjmi.com

How to cite this article: Corthouts P, Boels F, Van de Casteele E, Nadjmi N. Effects of various surgical protocols on maxillofacial growth in patients with unilateral cleft lip and palate: a systematic review. Plast Aesthet Res 2020;7:46.

http://dx.doi.org/10.20517/2347-9264.2020.97

\author{
Received: 28 Apr 2020 First Decision: 6 May 2020 Revised: 8 Jun 2020 Accepted: 24 Jun 2020 Published: 21 Aug 2020 \\ Academic Editor: Carroll Ann Trotman Copy Editor: Cai-Hong Wang Production Editor: Jing Yu
}

\begin{abstract}
Aim: The purpose of this study was to ascertain the effect of surgical procedures and their timing on maxillofacial growth in unilateral cleft lip and palate (UCLP) patients through a systematic literature review.
\end{abstract}

Methods: In December 2019, a search was conducted in PubMed and Web of Science on the basis of the keywords: "UCLP", "maxillofacial growth" and "facial growth", complemented by a hand search.

Results: Eleven articles were included. An important finding was the wide range of treatment protocols. Eight studies performed a multistage procedure, whereas three studies applied a simultaneous repair of cleft lip, palate, and alveolus in a single surgical session. The findings in these articles were based on cephalometric measures. Comparative tables were constructed regarding method of study and time and technique of closure.

Conclusion: The results of the articles were conflicting, and it was clear that more research on this subject is necessary. Overall, most studies agreed on the important factor of palatoplasty in maxillofacial growth. The most common finding was a retrusive maxillary growth in comparison to a noncleft control group. This was illustrated by a negative effect on A-point - nasion - B-point. A lot of discussion remains on the effect of lip closure. However, 
most studies seemed to agree that lip closure results in retro-inclined upper incisors. In conclusion, it is essential that an agreement be reached on the treatment for UCLP, since this is the most common congenital craniofacial condition.

Keywords: Unilateral cleft lip and palate, facial growth, maxillofacial growth

\section{INTRODUCTION}

Cleft lip and/or palate is one of the most common congenital malformations, it occurs in about 1 in 700 children $^{[1,2]}$. This malformation is due to failure in merging the facial processes at the correct time, which normally happens between week 7 and week 12 of gestation ${ }^{[1,3]}$. The etiology of unilateral cleft lip and palate (UCLP) is still not completely clear, it is definitely due to multiple factors and it is assumed to be caused by a combination of genetic and environmental aspects. Treatment of UCLP requires a multidisciplinary approach and a longitudinal follow-up. The team may consist of a maxillofacial surgeon, plastic surgeon, pediatrician, otolaryngologist, geneticist, orthodontist, dentist, psychologist, speech-language pathologist and audiologist. Because of the modern abilities of prenatal screening, UCLP can now already be detected early in gestation.

In patients with repaired UCLP, maxillofacial growth is often disturbed due to iatrogenic scar tissue caused by surgical closure of a cleft ${ }^{[4]}$. A retrusive midfacial region is characteristic of this population and becomes more obvious with age. Sagittal deficiency of the midface resulting in a concave facial profile is the most prominent feature seen in adult UCLP patients with disturbed maxillofacial growth ${ }^{[3]}$. However, in unoperated UCLP patients, midfacial growth is comparable to that in healthy, noncleft children without apparent restriction of growth ${ }^{[2]}$. Therefore an important objective is to restrict the iatrogenic impact of cleft surgery on midfacial growth ${ }^{[4]}$. According to the Eurocleft study, there are 194 different protocols for the treatment of UCLP ${ }^{[5]}$. The most controversial issues in the management of cleft palate are the timing of surgical intervention, speech development after various surgical procedures and the effects of surgery on facial growth ${ }^{[6]}$. UCLP and its treatment can affect the aesthetics, speech, and way of eating and chewing of a patient. A balance has to be found between these aspects to improve the child's quality of life.

The purpose of this review was to summarize the knowledge on the effect of different surgical protocols and surgical timing on maxillofacial growth. At this moment, there is no clear overview of all independent studies. The aim was to determine which timing and surgical approach is associated with the best results in this field.

\section{METHODS}

\section{Eligibility criteria}

This systematic review focused on UCLP patients and their treatment, more precisely the timing of surgical protocols and their effect on maxillofacial growth. For selection of the articles, the following inclusion criteria were applied: non-syndromic UCLP patient population; study population had to be over 6 years old; timing of each surgical procedure had to be known; no orthodontic procedures or orthognathic surgery was performed in the study population after surgical repair and before assessment; a control or comparison group had to be present, the outcome of the article had to be on maxillofacial growth; and evaluation of growth had to be at least based on a cephalogram. From the literature, it was clear that a great variety of landmarks were used in the different studies. The outcome parameters for this review were based on the maxillary position given by the sella-nasion-A-point angle (SNA), the mandibular position given by the sella-nasion-B-point angle ( $\mathrm{SNB}$ ) and the intermaxillary relationship [A-point-nasion-B-point angle (ANB)]. Articles had to provide measurements for at least SNA to be included in this review. Articles were 
excluded in which other forms of cleft lip and/or palate (cleft lip without cleft palate, cleft palate without cleft lip, bilateral cleft lip and palate) were analyzed. Furthermore, the following studies were excluded: case reports, literature reviews, systematic reviews and meta-analysis. Studies included in this review had to be written in English or Dutch and published after 2005. The goal of this systematic review was to compare various surgical protocols and to conclude which surgical protocol provides the most benefits regarding maxillofacial growth, functionality and aesthetics in UCLP patients of 6 years and older. Factors that would be assessed included: age of assessment, use of presurgical orthopedics, surgical timing, surgical approach (one-stage surgery, one-stage palatoplasty and two-stage palatoplasty), and ethnicity.

\section{Information sources}

A search of PUBMED and Web of Science was conducted in December 2019. The keywords used were "UCLP" and "Maxillofacial growth" or "Facial growth". As a search limit, the publication date was set to 2005 or later. The results obtained were stored in a single database (EndNote X9; Thomson Reuters, Philadelphia, USA). Additionally, a hand search of references of included articles in this systematic review was performed.

\section{Study selection}

After removal of duplicates, all articles were screened on title and abstract. This was performed independently by two authors to augment reliability. Any disparity in selected articles was discussed until a consensus was reached. Full-text articles were analyzed regarding our aforementioned inclusion and exclusion criteria. The articles used in the systematic reviews and literature review found through PubMed and Google Scholar were hand searched. The same protocol and eligibility criteria were applied as described above.

\section{Quality assessment and level of evidence}

The included articles were reviewed for good quality based on a checklist adopted from Liao et al. ${ }^{[7]}$. This checklist was adjusted for our review on the basis of the theory of Greenhalgh ${ }^{[8]}$ and can be found in the left column of Table $1^{[6,9-18]}$. Studies were considered to be of adequate size if their study population exceeded 100 people; this number was based on the quality assessment applied in the systematic review of Liao et al. ${ }^{[7]}$. Using the Oxford Centre for Evidence-Based Medicine 2011 v2.1 the qualified articles were assessed for their level of evidence by two independent reviewers. The levels could be downgraded on the basis of study quality, imprecision, indirectness, or inconsistency between studies, or because the effect size was very small. Disagreements were discussed until the two reviewers gave their consent.

\section{Data extraction}

From the final selection of articles, the following information was retrieved: author(s), publication year, study design, population studied, identification of the study groups, number of patients per group, mean age at time of assessment of facial growth, presurgical orthopedics (yes/no), lip closure technique and timing, soft palate closure technique and timing, hard palate closure technique and timing, alveolar cleft closure technique and timing, and final conclusions. The data were extracted from each article by one author and then checked by the second author before being collected in a database. Disagreements were resolved by discussion of each article to reach a consensus.

\section{RESULTS}

\section{Study selection}

The process of data collection and selection is shown in Figure 1. A total of 314 records were found among the databases investigated and 79 additional records, published after 2005, were identified through hand search of the articles included in this systematic review or provided by specialists on the subjects. After removal of duplicates $(n=103), 290$ articles remained that had their titles and abstracts assessed in 
Page 4 of 15

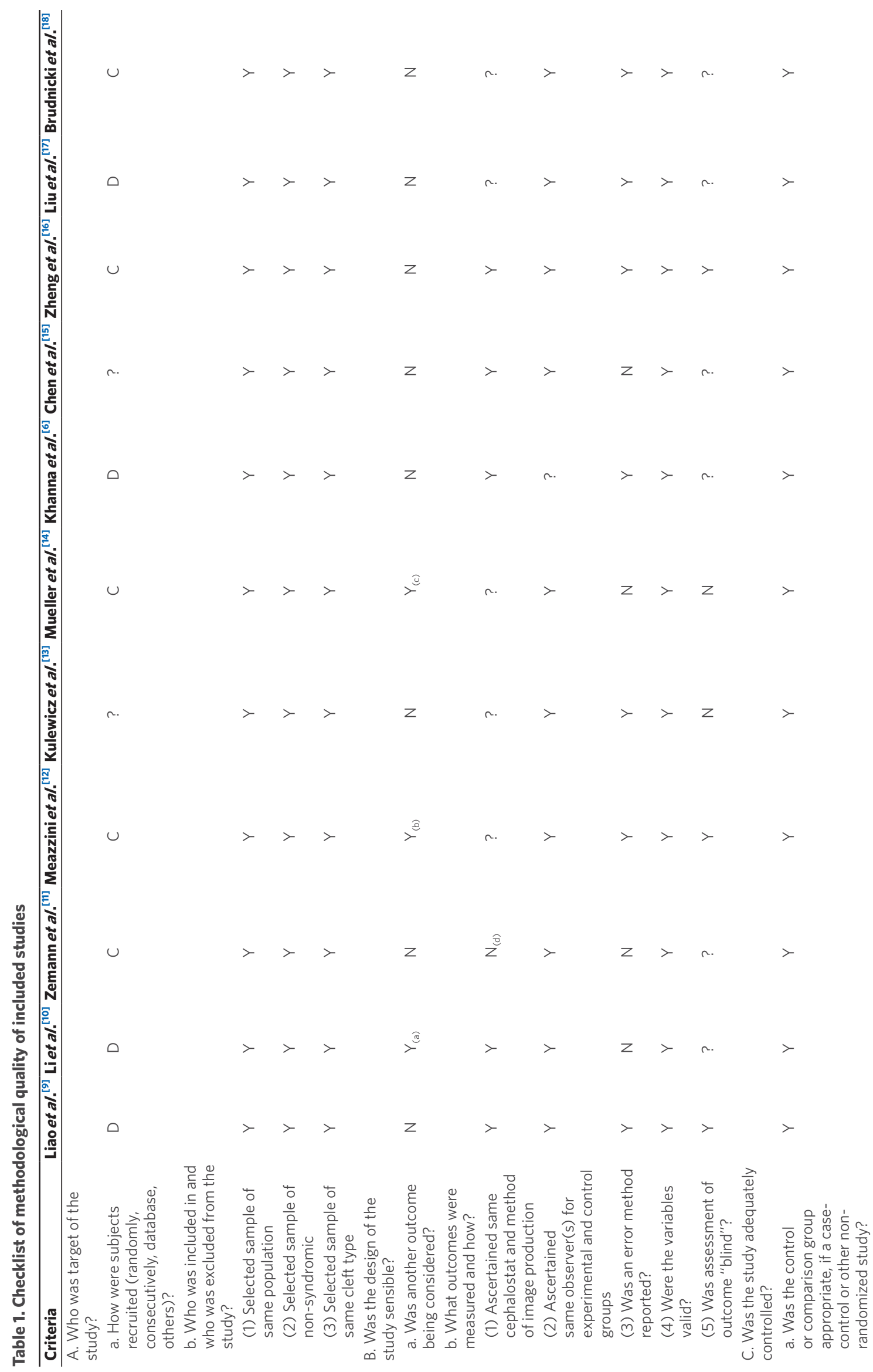




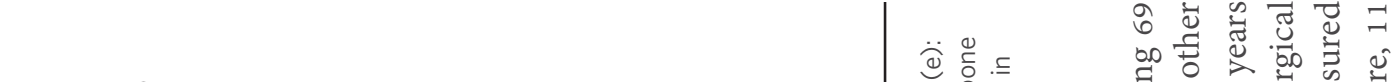

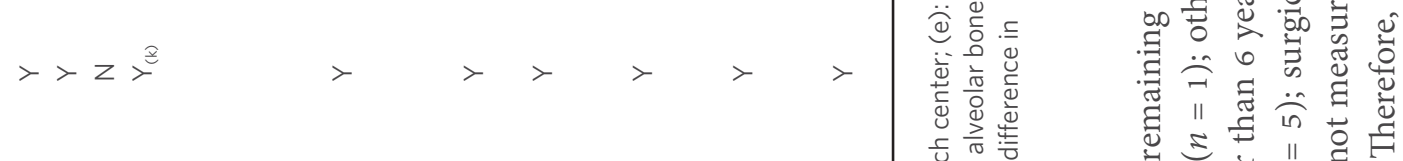

$$
\begin{aligned}
& >>\stackrel{E}{Z}> \\
& >>>>
\end{aligned}
$$

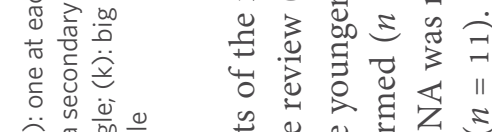

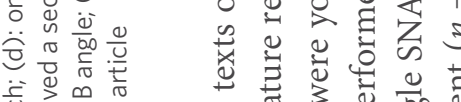

$$
\begin{aligned}
& \text { 它. } \\
& >>z \\
& >\quad>\quad z \quad>\quad z \\
& >>z \\
& \text { z } \\
& >\sim>^{\text {हิ }} \\
& >>z \\
& \text { z } \\
& >>z^{\widehat{e}} z \\
& >>\sim \cdot z \\
& >>>>^{\infty} \\
& >>>>\quad>\quad>
\end{aligned}
$$

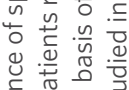

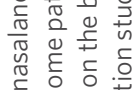

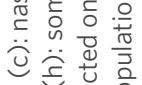

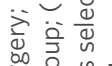

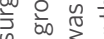

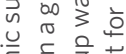

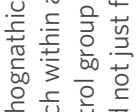

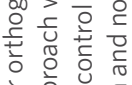

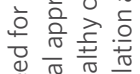

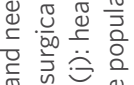

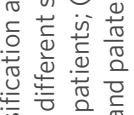

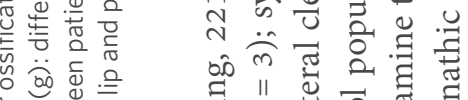

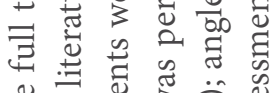

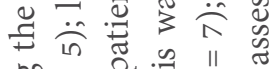

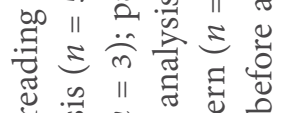

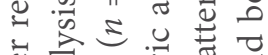

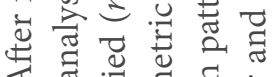

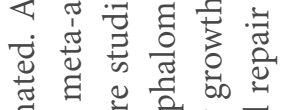

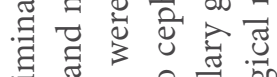

$$
\begin{aligned}
& \text { 寻 }
\end{aligned}
$$

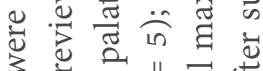

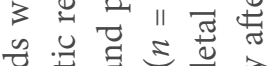

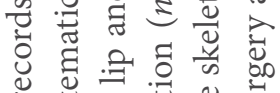

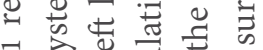

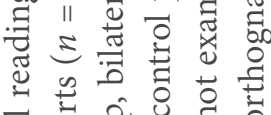

$$
\begin{aligned}
& \text { 클유유 }
\end{aligned}
$$

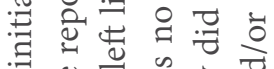

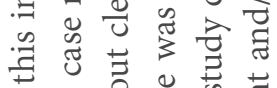

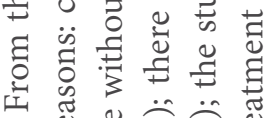

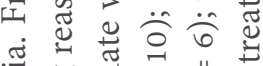

$$
\begin{aligned}
& \text { 离 } \\
& \text { 드응 }
\end{aligned}
$$

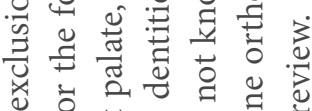

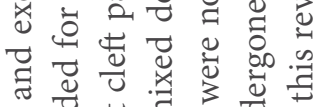

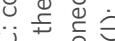

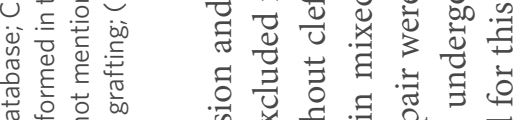

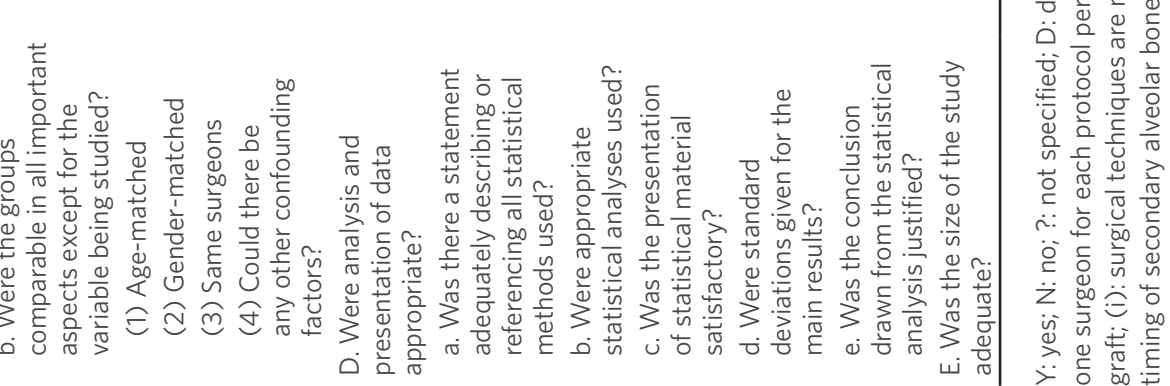

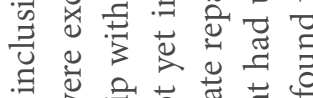

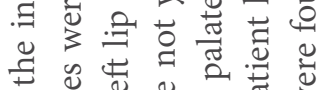

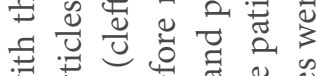

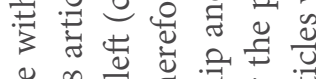

$$
\begin{aligned}
& \text { U艹 }
\end{aligned}
$$

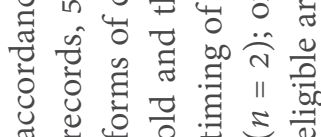




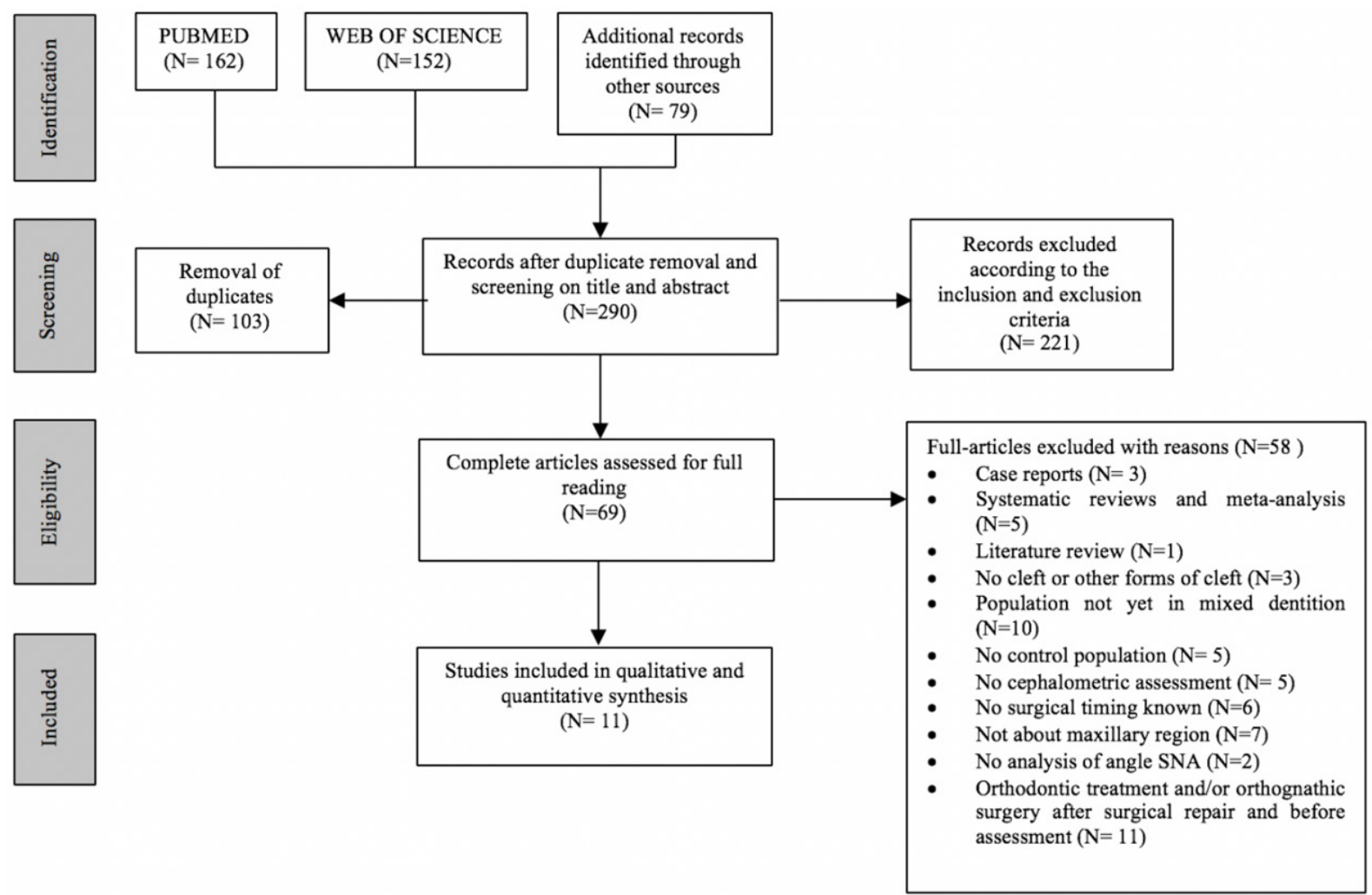

Figure 1. Flow chart showing the number of records identified and removed at each stage of the review. SNA: sella-nasion-A-point angle

\section{Study characteristics}

Most studies included in this review (73\%) were published after $2010^{[6,12-18]}$ [Table 2]. Six studies were conducted in Asia (55\%) and 5 were conducted in Europe (45\%). The variety of treatment protocols that were administered in the different studies in the articles, as well as whether or not presurgical orthopedics was performed, is shown in Table 2. Sample sizes consisted of 10 subjects $^{[12]}$ to 128 subjects $^{[18]}$. Seven articles included a healthy, noncleft control group in their study ${ }^{[10,11,13-17]}$. The authors used a variety of surgical techniques. Regarding cheiloplasty, the following techniques were used: (modified) Millard rotationadvancement technique ${ }^{[9-12,15,16]}$, triangular technique (by Tennison with modifications $)^{[9,11,13,18]}$ and modified Delaire technique ${ }^{[12]}$. Three articles did not specify the technique used for lip repair ${ }^{[6,14,17]}$. Concerning closure of the palate several techniques are listed, which can be categorized as follows: (modified) von Langenbeck ${ }^{[9,12,13]}$, pushback palatoplasty ${ }^{[10]}$, cranial or caudally pedicled vomer flap ${ }^{[9,12-14,18]}$, intravelar veloplasty ${ }^{[11]}$, modified Pigott technique ${ }^{[12]}$ and two-flap palatoplasty ${ }^{[14-16]}$. Khanna et al. ${ }^{[6]}$ provided no description of the technique used for palate repair.

Three different surgical techniques were described for alveolar cleft closure: gingivoperiosteoplasty ${ }^{[11,12]}$, primary bone grafting ${ }^{[14]}$ and secondary bone grafting ${ }^{[11,12,16,18]}$. Three studies declared that the patients in the samples had not undergone bone grafting surgery ${ }^{[9,10,17]}$, whereas three studies had no information on whether or not bone grafting surgery had been done $e^{[6,13,15]}$.

The mean age at the time of surgical repair in patients with repaired UCLP varied according to the surgical protocol: lip repair before 15 weeks $^{[6]}$ until 2 years ${ }^{[15]}$; soft palate closure from 4 months $^{[12]}$ to 5 years ${ }^{[9]}$; hard palate closure from 3 months ${ }^{[12]}$ to 4 years ${ }^{[9]}$ and alveolar cleft repair from 6 months ${ }^{[14]}$ to 11 years ${ }^{[12,16]}$.

In 3 studies, the surgical protocol consisted of the primary one-stage surgery of UCLP: simultaneous repair 


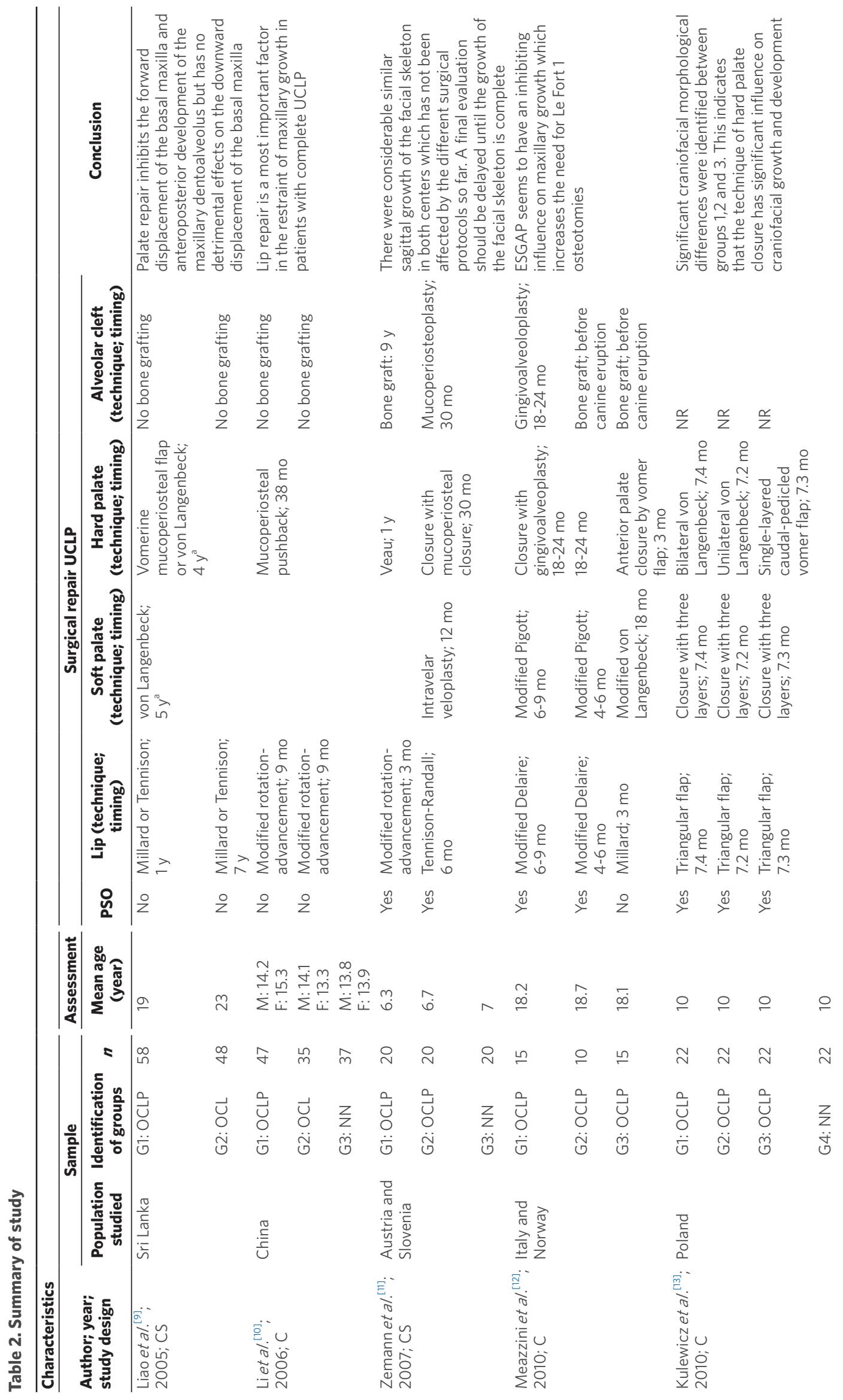




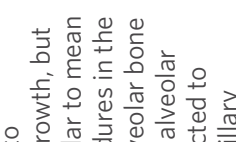

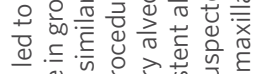

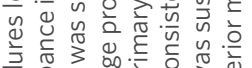

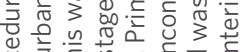

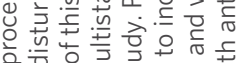
$\begin{array}{lllll} & \\ 0\end{array}$ $\begin{array}{llll} & \\ 0 & \end{array}$

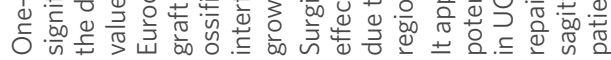

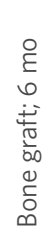

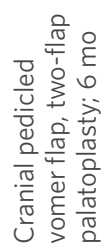

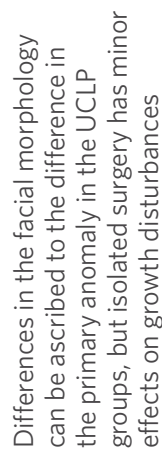

ำำ
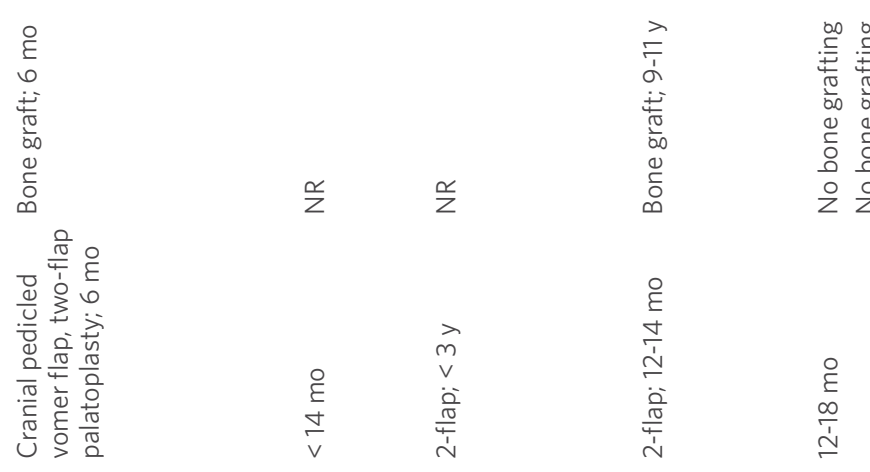

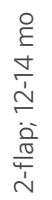

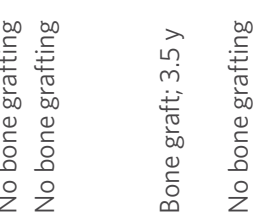

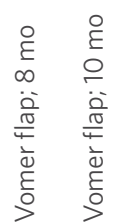
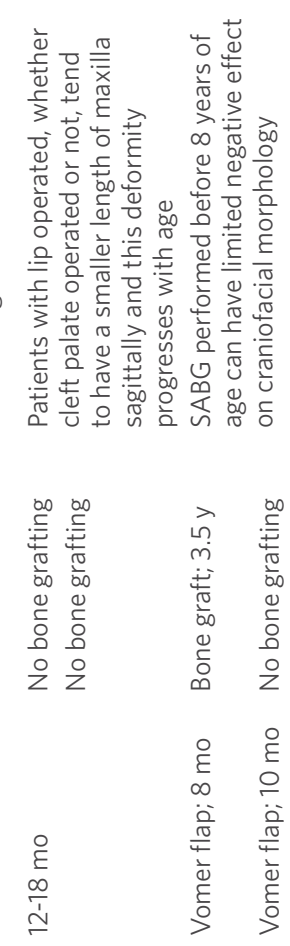

$\stackrel{\circ}{\xi}$

$\stackrel{\circ}{\natural}$

品

$a m \quad m o v$

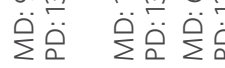

ㅇํํ $\stackrel{m}{m}$

ำร

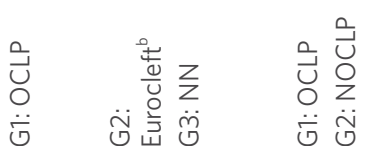

m

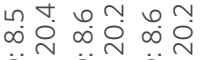

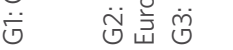

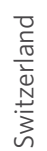

$\stackrel{-\frac{\pi}{\bar{c}}}{\underline{\underline{\underline{x}}}}$

:

$\frac{d}{e^{2}}$

ฮั

产

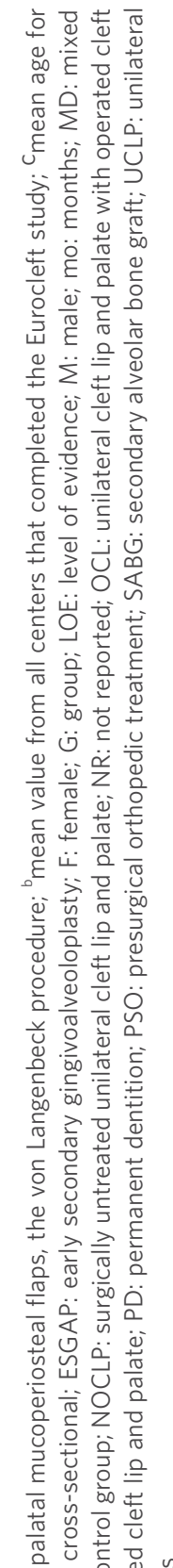

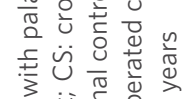

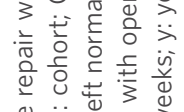

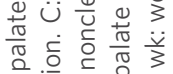

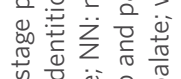

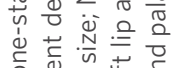

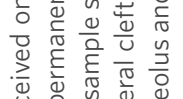

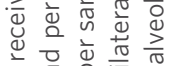

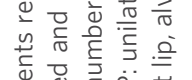

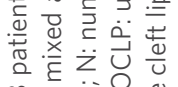

等
تี

范

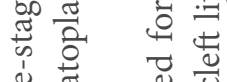

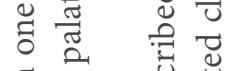

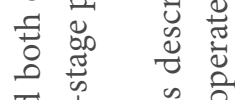

च

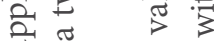

के

龸芯

क ज्ञ 유

리 결

चै के ठै

E

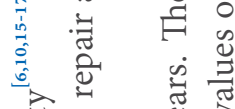

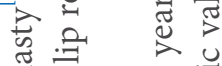

맘

节茕

莽 듕

कँ

ปี

प्रे के

है की

\& 원 त्ञ

节苛苛

क त्ञ

究 흥

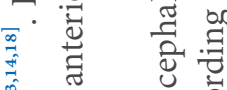

해

范

तु

$\exists$ ठ

क

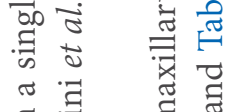

: 고

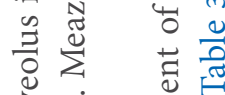

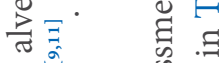

चี

है ने है

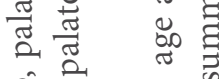

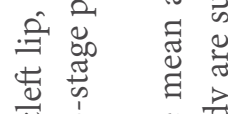

范它 
Table 3. Comparison of cephalometric values of each study with UCLP patients in mixed dentition (6-12 y), classified according to study population. Specification of groups can be found in Table 2

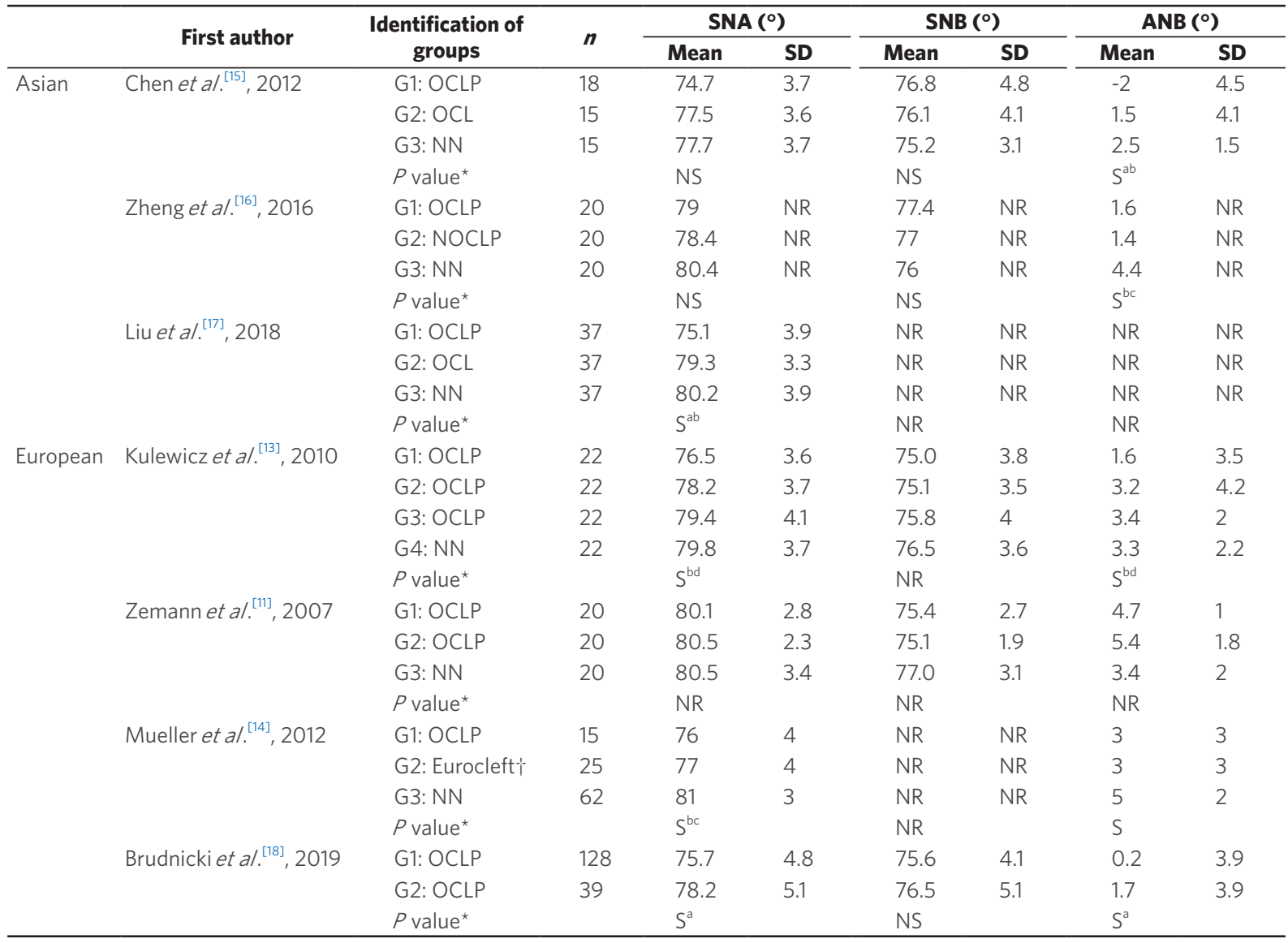

$\dagger$ Mean value from all centers that completed the Eurocleft study; ${ }^{\mathrm{a} G 1-G 2:} P$-value $<0.05 ;{ }^{\mathrm{b}} \mathrm{G} 1-\mathrm{G} 3: P$-value $<0.05 ;{ }^{\mathrm{C}} \mathrm{G} 2$-G3: $P$-value $<$ $0.05 ;{ }^{\mathrm{d}} \mathrm{G} 1-\mathrm{G} 4: P$-value $<0.05$. ${ }^{\star} P$-value $<0.05$ was regarded as significant. Comparisons between groups are mentioned only when undertaken in the study and regarded as significant. G: group; F: female; M: male; n: number of sample size; NN: noncleft normal control group; NOCLP: surgically untreated unilateral cleft lip and palate; NR: not reported; NS: not significant; OCL: unilateral cleft lip and palate with operated cleft lip only; OCLP: unilateral cleft lip and palate with operated cleft lip and palate; UCLP: unilateral cleft lip and palate; S: significant; SD: standard deviation; y: years

palate (OCLP) in each study were compared with the following groups: UCLP patients treated according to a different protocol ${ }^{[11-13,18]}$; UCLP patients with operated cleft lip and unoperated cleft palate ${ }^{[9,10,15,17]}$; and non-treated UCLP patients ${ }^{[6,16]}$, mean value from all centers that completed the Eurocleft study ${ }^{[14]}$. In seven studies ${ }^{[10,11,13-17]}$ noncleft children served as normal controls. Among the abovementioned groups, 10 of the 11 included articles ${ }^{[6,9-11,13-18]}$ reported a $P$-value less than 0.05 for one or more of the cephalometric values SNA, SNB and ANB, whereas one study ${ }^{[12]}$ did not report a corresponding $P$-value. A $P$-value less than 0.05 was regarded as significant.

\section{Quality assessment and level of evidence}

The methodological quality of the 11 articles was evaluated using the aforementioned checklist, which can be seen in Table 1. None of the included articles were of perfect methodological quality, they showed different deficiencies, but overall, they were deemed of good quality. Only 4 studies ${ }^{[9,10,17,18]}$ were deemed large enough, this showing that there is a need for more research with a substantial study population. All included studies were retrospective (level 3 evidence).

\section{Surgical repair and maxillofacial growth}

Six out of 11 included articles evaluated the effect that surgery itself has on maxillofacial growth in children with UCLP ${ }^{[6,9,10,15-17]}$. Khanna et al. ${ }^{[6]}$ compared a group of treated UCLP patients with a group of non- 
Table 4. Comparison of cephalometric values of each study with UCLP patients in permanent dentition (12-23 y), classified according to study population. Specification of groups can be found in Table 2

\begin{tabular}{|c|c|c|c|c|c|c|c|c|c|}
\hline & \multirow{2}{*}{ Authors } & \multirow{2}{*}{$\begin{array}{l}\text { Identification of } \\
\text { groups }\end{array}$} & \multirow{2}{*}{$n$} & \multicolumn{2}{|c|}{ SNA $\left(^{\circ}\right)$} & \multicolumn{2}{|c|}{ SNB $\left(^{\circ}\right)$} & \multicolumn{2}{|c|}{ ANB ( $\left(^{\circ}\right)$} \\
\hline & & & & Mean & SD & Mean & SD & Mean & SD \\
\hline \multirow[t]{18}{*}{ Asian } & Liao et al. ${ }^{[9]}, 2005$ & G1: OCLP & 58 & 79.6 & NR & 78.3 & NR & 1.4 & NR \\
\hline & & G2: OCL & 48 & 83.0 & NR & 78.6 & NR & 4.4 & NR \\
\hline & & $P$ value ${ }^{*}$ & & $S^{a}$ & & NS & & $S^{a}$ & \\
\hline & Li et $a l^{[10]}, 2006$ & G1: OCLP (M/F) & 47 & $73.5 / 73.2$ & $4.7 / 6.8$ & $74.8 / 75.8$ & $4.7 / 4.9$ & $-1.3 /-2.6$ & $2.8 / 3.9$ \\
\hline & & G2: OCL (M/F) & 35 & $72.6 / 75.1$ & $5.3 / 3.4$ & $73.3 / 75.2$ & $4.3 / 6.0$ & $-0.6 /-0.0$ & $4.0 / 4.2$ \\
\hline & & G3: NN (M/F) & 37 & $82.1 / 80.3$ & $2.6 / 3.2$ & $78.5 / 77.4$ & $2.5 / 3.0$ & $3.6 / 2.9$ & $2.2 / 1.3$ \\
\hline & & $P$ value $(M / F)^{\star}$ & & $\mathrm{S}^{\mathrm{bc}} / \mathrm{S}^{\mathrm{bc}}$ & & $\mathrm{S}^{\mathrm{bc}} / \mathrm{NS}$ & & $\mathrm{S}^{\mathrm{bc}} / \mathrm{S}^{\mathrm{abc}}$ & \\
\hline & Khanna et al. ${ }^{[6]}, 2012$ & G1: OCLP & 25 & 73.2 & 13.9 & NR & NR & NR & NR \\
\hline & & G2: NOCLP & 47 & 83.6 & 4.3 & NR & NR & NR & NR \\
\hline & & $P$ value ${ }^{\star}$ & & $S^{a}$ & & NR & & NR & \\
\hline & Chen et $a l_{.}^{[15]}, 2012$ & G1: OCLP & 15 & 75.5 & 6.6 & 79.7 & 6.4 & -4.2 & 5.1 \\
\hline & & G2: OCL & 15 & 79.3 & 4.9 & 79 & 3.3 & 0.3 & 4.4 \\
\hline & & G3: NN & 15 & 80.6 & 3.0 & 77.2 & 2.9 & 3.4 & 1.9 \\
\hline & & $P$ value ${ }^{*}$ & & $S^{b}$ & & NS & & $\mathrm{S}^{\mathrm{abc}}$ & \\
\hline & Liu et $a]_{.}^{[17]}, 2018$ & G1: OCLP & 37 & 75.8 & 5.1 & NR & NR & NR & NR \\
\hline & & G2: OCL & 37 & 77.3 & 4.8 & NR & NR & NR & NR \\
\hline & & G3: NN & 37 & 81.7 & 2.9 & NR & NR & NR & NR \\
\hline & & $P$ value ${ }^{*}$ & & $S^{b c}$ & & NR & & NR & \\
\hline \multirow[t]{8}{*}{ European } & Meazzini et al. ${ }^{[12]}, 2010$ & G1: OCLP & 15 & 74.9 & 3.5 & 76.9 & 3.0 & -1.9 & 2.7 \\
\hline & & G2: OCLP & 10 & 76.7 & 3.3 & 77.4 & 2.6 & -0.8 & 3.3 \\
\hline & & G3: OCLP & 15 & 75.8 & 3.5 & 77.1 & 4.3 & -1.3 & 1.9 \\
\hline & & $P$ value ${ }^{*}$ & & NR & & NR & & NR & \\
\hline & Mueller et al. ${ }^{[14]}, 2012$ & G1: OCLP & 7 & 76 & 4 & NR & NR & -0.2 & 3 \\
\hline & & G2: Eurocleft $†$ & 25 & 75 & 4 & NR & NR & 0.9 & 3 \\
\hline & & G3: NN & 71 & 81 & 4 & NR & NR & 4 & 2 \\
\hline & & $P$ value* & & $S^{b c}$ & & NR & & $S^{b c}$ & \\
\hline
\end{tabular}

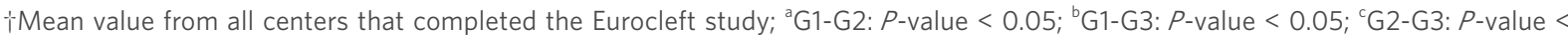
0.05. ${ }^{\star} P$-value $<0.05$ was regarded as significant. Comparisons between groups are mentioned only when undertaken in the study and regarded as significant. G: group; F: female; M: male; n: number of sample size; NN: noncleft normal control group; NOCLP: surgically untreated unilateral cleft lip and palate; NR: not reported; NS: not significant; OCL: unilateral cleft lip and palate with operated cleft lip only; OCLP: unilateral cleft lip and palate with operated cleft lip and palate; UCLP: unilateral cleft lip and palate; S: significant; SD: standard deviation; y: years

treated UCLP patients between the age of 12 and 20 years old. They found different values by comparing the cephalometric measurements of the two groups, and they concluded that surgical intervention does interfere with growth in the facial region due to scar tissue in the lip and palate.

Four articles identified the effects of palate repair on maxillary morphology ${ }^{[9,10,15,17]}$. These studies recruited patients with non-syndromic UCLP who had lip repair only (OCL) and patients with non-syndromic UCLP who had lip and palate repairs (OCLP). Palate repair at an early stage in patients with UCLP seems to result, in the long run, in a larger retrusion of the maxilla (SNA) and smaller anteroposterior jaw relation (ANB) than in the OCL group, who demonstrated an almost normal maxillary growth ${ }^{[9,15,17]}$. Opposed to this view, Li et al ${ }^{[10]}$ reported a smaller SNA angle in both OCL and OCLP groups than the normal control group and concluded that lip repair is primarily responsible for the midfacial hypodevelopment in cleft patients.

However, Zheng et al. ${ }^{[16]}$ attributes the difference in cephalometric results to the intrinsic effect of UCLP on the maxilla resulting in a developmental deficiency and claims that surgery has minor effects on growth disturbances. They discovered that the tendency in patients with UCLP (with or without surgical repair) toward a less protruded alveolar maxilla (SNA) and a more protruded alveolar mandible (SNB) gave rise to the low anteroposterior jaw relation at the alveolar level (ANB). 


\section{Surgical technique and maxillofacial growth}

Five of the 11 included articles evaluated the effect of different surgical techniques and protocols on maxillofacial growth in children with UCLP ${ }^{[11-14,18]}$. Three of the 11 studies looked into the implementation of a one-stage surgery and compared their results with a healthy control population ${ }^{[1,13,14]}$. Considering the age at the time of assessment in these 3 studies, the results concerning sagittal growth were very diversified when comparing the outcomes. The study of Zemann et al. ${ }^{[11]}$ showed no significant difference regarding the angles SNA, SNB and ANB at the age of 6 years old when comparing patients treated according to various one-stage protocols. Furthermore, the acquired values were equivalent to those in a healthy control group. However, Mueller et al. ${ }^{[14]}$ concluded that maxillary protrusion (SNA) and anteroposterior jaw relation (ANB) in the one-stage groups differed significantly from those of the noncleft, healthy control group, but the degree of disturbance in growth was similar to mean values of multistage approaches in the Eurocleft study. Kulewicz et al. ${ }^{[13]}$ conducted comparative research into 3 different techniques of palate repair applied to a one-stage surgical approach and checked this against healthy controls. Cephalometric parameter comparison analysis demonstrated significant differences between the 4 groups regarding maxillary prominence (SNA) and maxillo-mandibular relationship (ANB). This indicates that the technique of hard palate closure has a substantial influence on maxillofacial growth and development.

Meazzini et al. ${ }^{[12]}$ did a comparison between UCLP patients treated with 3 different protocols to evaluate the long-term results between closure of the hard palate at 18-36 months together with early secondary gingivoalveoloplasty (ESGAP) and alveolar cleft repair at 9-11 years of age. Using a longitudinal cephalometric evaluation, they found that patients who underwent ESGAP had a decreased maxillary prominence (SNA) and showed an inhibition of maxillary growth compared with the 2 secondary bone graft groups, while mandibular prominence (SNB) increased in the 3 groups. Nonetheless, performing alveolar bone grafting before 8 years of age is suspected to interfere with anterior maxillary growth, and the timing of bone grafting can be essential to maxillofacial growth. Studies suggested that performing the surgery at a later age would prove the most beneficial ${ }^{[14,18]}$.

\section{DISCUSSION}

Maxillary growth in UCLP patients has already been widely addressed in the literature; nevertheless, a wide variation in results was found. More often than not, no consensus was reached relating to the vertical and anteroposterior growth pattern in UCLP patients. On the one hand, they propose that there may be a potentially normal maxillary growth in untreated UCLP patients ${ }^{[15]}$, and on the other hand, they propose that regardless of the treatment, UCLP patients show retrusion of the maxilla and decreased maxillary length, where there are many causes to be considered. Some reports attribute this retrusion to the intrinsic defect of the cleft ${ }^{[16,17]}$, while others claim it is from surgical intervention ${ }^{[6,13-15,19]}$ and even dependent on the skill of the surgeon. The disturbing effect on the growth of the maxillary skeleton after surgical repair is due to devascularization, disturbance of the periosteum or the restrictive effect of the scar ${ }^{[16]}$. Therefore, surgery leads to maxillary hypoplasia: the maxillary angle (SNA) and the maxillomandibular angle (ANB) were smaller and negative when matched to the normal population ${ }^{[19]}$. Unoperated cleft patients had a more favorable morphology of craniofacial structures when compared with surgically treated patients, indicating that due to alteration of the peri-oral functional matrix, surgical intervention interferes with the growth process in UCLP patients. They point to the scar tissue in lip and palate region being the factor due to its restraining effect on maxillofacial growth. The alterations in these functional matrices are important in determining the growth of facial structures. Moreover, maxilla length was found to be significantly reduced in surgically treated UCLP patients, and they showed a significant reduction in cranial base angle ${ }^{[6]}$.

Without doubt, palate closure is the most documented part of the treatment protocol for UCLP. Many surgical protocols exist, using different techniques and surgical timings and have been evaluated in terms of benefits to maxillary growth, speech development, velopharyngeal function and quality of life. An 
important objective is to reduce the number of operations as they are considered to be stressful for the family and to make it more difficult to cope successfully. Likewise, the number of surgeries has an impact on the psychological well-being of the patient. There does not seem to be any consensus on the best time to perform palate closure, where every timing has its own advantages and disadvantages ${ }^{[7,20]}$. Some studies ${ }^{[15,19,21,22]}$ assumed that the early surgical repair of the cleft palate is responsible for the impaired maxillary growth and concluded that it was better to delay surgical palate repair. During the maxillary growth spurt an important proportion of the final length of the maxilla is gained. It is possible that the benefit of delayed hard palate on maxillofacial growth closure can only be achieved by closing when the greatest proportion of the final maxillary length is already achieved ${ }^{[19]}$. However, Zheng et al. ${ }^{[16]}$ claim that isolated surgery has minor effects on growth disturbances and conclude that early palatal closure should therefore be performed because it will not negatively affect maxillofacial growth. Furthermore, early primary repair operations facilitate ease of feeding and good speech development, and there is a strong desire from the patient's parents themselves to have the cleft closed as early as possible ${ }^{[7,22,23]}$. Nevertheless, the growth spurt of these children should be awaited before conclusive results are formed concerning the measured cephalometric values regarding sagittal growth of the skeleton, since the results in patients in mixed dentition show a lot of variability. Regarding this concept, researchers should be aware of the fact that the end of growth in cleft children is later than in healthy noncleft children ${ }^{[24]}$.

Whereas most studies agree that palatal closure is the most detrimental factor for the evolution of maxillary growth, other studies are convinced that lip repair is the most important factor in the restraint of maxillary growth in patients with UCLP ${ }^{[10,25]}$. There is however agreement that pressure from a tense upper lip causes retro inclined upper incisors, a retruded maxilla and obtuse nasolabial angle ${ }^{[26]}$. This usually results in an anterior cross bite ${ }^{[2]}$. It is crucial to stretch the importance of an optimal result of lip closure. Lip, nose and chin are the key regions in a patient's face and they have the most significant impact on facial aesthetics, self-esteem and self-image. Thus lip, nose and columella ${ }^{[27]}$ are most frequently surgically revised in UCLP patients.

There is still a lot of discussion about which technique and timing is most beneficial for alveolar closure. Alveoloplasty is performed to stabilize the maxillary arch, facilitate the eruption of the canine (and the lateral incisor), raise the alar base of the nose and to reconstruct the residual nasoalveolar fistula ${ }^{[28]}$. Overall, 3 used techniques can be distinguished ${ }^{[2]}$ : gingivoperiosteoplasty, primary bone grafting and secondary bone grafting. Although gingivoperiosteoplasty has the big advantage that it requires fewer surgeries, it seemed to have an inhibitory effect on maxillary growth ${ }^{[29]}$. Primary bone grafting led to inconsistent alveolar ossification and was suspected to interfere with anterior maxillary growth ${ }^{[14]}$. Patients treated with secondary bone grafting seemed to have better maxillary growth and appeared to be needing less orthognathic surgery ${ }^{[29]}$. Brudnicki et al. ${ }^{[18]}$ discovered that maxillary length increased when alveolar bone grafting was performed at a later age, specifically when performed beyond the age of eight years old. This would suggest that the timing of bone grafting is critical to maxillofacial growth.

Unanimity with regard to a superior treatment protocol in terms of closure of the lip, closure of the palate and closure of the alveolar cleft, was not reached in this systematic review. The reasons for conflicting results from the selected studies include the great variance in treatment protocols, as shown by the varied timing of surgical repair and different surgical techniques [Table 2]. This systematic review also had some methodological deficiencies [Table 1] and limitations. First, 4 studies ${ }^{[6,9,12,18]}$ did not compare operated UCLP patients with a noncleft control group. Consequently, it is not clear how the measured cephalometric outcomes are related to a healthy, normal population. Second, some studies were well designed and wellexecuted but had small sample sizes. Seven ${ }^{[6,11-16]}$ of the 11 included articles had samples less than 100 patients. This could imply that the statistical power of these studies was too low to detect differences. Third, one study ${ }^{[10]}$ examined the cephalometric values for males and females separately and this might have 
resulted in an analysis bias, whereas another study ${ }^{[12]}$ did not provide a corresponding $\mathrm{p}$-value for their cephalometric outcomes. Fourth, the study population used in the different articles included in this review had a lot of ethnic diversity. Therefore, it is unsure if all findings apply to all different ethnical groups. It is important to take this into account when using the results of this systematic review. Fifth, none of the included studies had a level of evidence higher than 3. This means that there was a shortage of highquality randomized controlled trials on the effects of surgical timing and techniques on maxillofacial growth. To get more high-quality studies, follow-up of patients should be over a longer period of time. Preferably, patients should be followed starting from mixed dentition until after their growth spurt, ending at adult age. Sixth, detailed documentation of the study population, the technique for surgical cleft closure, number of surgeons, grade of surgeon and information whether orthodontic or orthognathic treatment was performed, were insufficiently described or lacking, making the studies unsuitable for meta-analysis. Hence, no attempt was made to perform pooled analysis, and the evidence was summarized qualitatively.

Future treatment research should be established with special attention towards methodology, well described study population, number of surgeons, grade of surgeon, technique of surgical closure and information on the undergoing of orthodontic or orthognathic treatments since early intervention may result in a better outcome. Kappen et al. ${ }^{[4]}$ proposed that a multidisciplinary and multicenter database of cleft children should be set up. If this would be the case, a prospective study could be conducted on these patients. This might help in the further determination of the best time of closure of both lip and palate. Consequently, on the basis of a study like this, a universal protocol might be possible for the treatment of cleft children to guarantee them the best results. Furthermore, they stressed the importance of calculating the burden on the caregivers as well as the costs of the procedures into the determination of the best protocol.

In conclusion, most studies agree that palatoplasty is the main factor attributing to disturbance of maxillofacial growth; in addition, it is crucial to limit the amount of postoperative scar tissue. In palatoplasty performed after the growth spurt, the maxillofacial growth is least affected. But studies also agree that it is important to find a balance between aesthetics, functionality and quality of life. Therefore, it is not recommended to perform palatoplasty only after the growth spurt despite of the better effect on maxillofacial growth because this impedes speech development too much.

There is however a consensus about the timing of lip closure. It has to be performed between three and six months of age. It is also widely accepted that lip closure could have a negative influence on maxillofacial growth.

From the studies on alveoloplasty, it can be concluded that secondary bone grafting has the most beneficial outcome on maxillofacial growth; however, when using gingivoperiosteoplasty, there is less need of a third surgery.

In the articles studied in this review the functional result of UCLP repair is considered to be more important than the aesthetic result. More studies still need to be conducted to ascertain the best timing of surgery and to design a technique that creates both optimal functional and aesthetic results to guarantee the well-being of the patient.

It is important to properly understand the causal factors that result in an impeded maxillary growth. This will help in enabling proper planning of treatment, minimizing orthodontic treatment time and in reducing major secondary corrective surgeries. All of this combined illustrates that in the treatment of UCLP, a longitudinal follow-up and a multidisciplinary approach are crucial. More studies still need to be conducted to make sure the best outcome can be acquired. 


\section{DECLARATIONS}

\section{Authors' contributions}

Made substantial contributions to conception and design of the study and performed data analysis and interpretation: Corthouts P, Boels F, Van de Casteele E, Nadjmi N

Performed data acquisition and provided administrative, technical, and material support as well: Corthouts P, Boels F, Van de Casteele E, Nadjmi N

Reviewed the manuscript for content and grammar/spelling mistakes: Corthouts P, Boels F, Van de Casteele E, Nadjmi N

\section{Availability of data and materials}

Not applicable.

\section{Financial support and sponsorship}

None.

\section{Conflicts of interest}

All authors declared that there are no conflicts of interest.

\section{Ethical approval and consent to participate}

Not applicable.

\section{Consent for publication}

Not applicable.

\section{Copyright}

(c) The Author(s) 2020.

\section{REFERENCES}

1. Mossey PA, Little J, Munger RG, Dixon MJ, Shaw WC. Cleft lip and palate. Lancet 2009;374:1773-85.

2. Farronato G, Kairyte L, Giannini L, Galbiati G, Maspero C. How various surgical protocols of the unilateral cleft lip and palate influence the facial growth and possible orthodontic problems? Which is the best timing of lip, palate and alveolus repair? literature review. Stomatologija 2014;16:53-60.

3. Goyenc YB, Gurel HG, Memili B. Craniofacial morphology in children with operated complete unilateral cleft lip and palate. J Craniofac Surg 2008;19:1396-401.

4. Kappen IFPM, Yoder WR, Mink van der Molen ABM, Breugem CC. Long-term craniofacial morphology in young adults treated for a non-syndromal UCLP: a systematic review. J Plast Reconstr Aesthet Surg 2018;71:504-17.

5. Shaw WC, Semb G, Nelson P, Brattstrom V, Molsted K, et al. The eurocleft project 1996-2000: overview. J Craniomaxillofac Surg 2001;29:131-40; discussion 41-2.

6. Khanna R, Tikku T, Wadhwa J. Nasomaxillary complex in size, position and orientation in surgically treated and untreated individuals with cleft lip and palate: a cephalometric overview. Indian J Plast Surg 2012;45:68-75.

7. Liao YF, Mars M. Hard palate repair timing and facial growth in cleft lip and palate: a systematic review. Cleft Palate Craniofac J 2006;43:563-70.

8. Greenhalgh T. Assessing the methodological quality of published papers. BMJ 1997;315:305-8.

9. Liao YF, Mars M. Long-term effects of palate repair on craniofacial morphology in patients with unilateral cleft lip and palate. Cleft Palate Craniofac J 2005;42:594-600.

10. Li Y, Shi B, Song QG, Zuo H, Zheng Q. Effects of lip repair on maxillary growth and facial soft tissue development in patients with a complete unilateral cleft of lip, alveolus and palate. J Craniomaxillofac Surg 2006;34:355-61.

11. Zemann W, Mossbock R, Karcher H, Kozelj V. Sagittal growth of the facial skeleton of 6-year-old children with a complete unilateral cleft of lip, alveolus and palate treated with two different protocols. J Craniomaxillofac Surg 2007;35:343-9.

12. Meazzini MC, Rossetti G, Garattini G, Semb G, Brusati R. Early secondary gingivo-alveolo-plasty in the treatment of unilateral cleft lip and palate patients: 20 years experience. J Craniomaxillofac Surg 2010;38:185-91.

13. Kulewicz M, Dudkiewicz Z. Craniofacial morphological outcome following treatment with three different surgical protocols for complete unilateral cleft lip and palate: a premilinary study. Int J Oral Maxillofac Surg 2010;39:122-8. 
14. Mueller AA, Zschokke I, Brand S, Hockenjos C, Zeilhofer HF, et al. One-stage cleft repair outcome at age 6- to 18-years - a comparison to the Eurocleft study data. Br J Oral Maxillofac Surg 2012;50:762-8.

15. Chen ZQ, Wu J, Chen RJ. Sagittal maxillary growth pattern in unilateral cleft lip and palate patients with unrepaired cleft palate. J Craniofac Surg 2012;23:491-3.

16. Zheng ZW, Fang YM, Lin CX. Isolated influences of surgery repair on maxillofacial growth in complete unilateral cleft lip and palate. J Oral Maxillofac Surg 2016;74:1649-57.

17. Liu X, Chen Z. Effects of palate repair on cranial base and maxillary morphology in patients with unilateral complete cleft lip and palate. Cleft Palate Craniofac J 2018;55:1367-74.

18. Brudnicki A, Sawicka E, Brudnicka R, Fudalej PS. Effects of different timing of alveolar bone graft on craniofacial morphology in unilateral cleft lip and palate. Cleft Palate Craniofac J 2020;57:105-13.

19. Kappen IFPM, Bittermann GKP, Schouten RM, Bittermann D, Etty E, et al. Long-term mid-facial growth of patients with a unilateral complete cleft of lip, alveolus and palate treated by two-stage palatoplasty: cephalometric analysis. Clin Oral Investig 2017;21:1801-10.

20. Salgado KR, Wendt AR, Fagundes NCF, Maia LC, Normando D, et al. Early or delayed palatoplasty in complete unilateral cleft lip and palate patients? A systematic review of the effects on maxillary growth. J Craniomaxillofac Surg 2019;47:1690-8.

21. Liao YF, Cole TJ, Mars M. Hard palate repair timing and facial growth in unilateral cleft lip and palate: a longitudinal study. Cleft Palate Craniofac J 2006;43:547-56.

22. Gopinath VK, Samsudin AR, Noor SNFM, Sharab HYM. Facial profile and maxillary arch dimensions in unilateral cleft lip and palate children in the mixed dentition stage. Eur J Dent 2017;11:76-82.

23. Holland S, Gabbay JS, Heller JB, O'Hara C, Hurwitz D, et al. Delayed closure of the hard palate leads to speech problems and deleterious maxillary growth. Plast Reconstr Surg 2007;119:1302-10.

24. Batwa W, Almoammar K, Aljohar A, Alhussein A, Almujel S, et al. The difference in cervical vertebral skeletal maturation between cleft lip/palate and non-cleft lip/palate orthodontic patients. Biomed Res Int 2018;2018:5405376.

25. Bichara LM, Araujo RC, Flores-Mir C, Normando D. Impact of primary palatoplasty on the maxillomandibular sagittal relationship in patients with unilateral cleft lip and palate: a systematic review and meta-analysis. Int J Oral Maxillofac Surg 2015;44:50-6.

26. Ebin LE, Zam NMZ, Othman SA. Cephalometric analysis of Malay children with and without unilateral cleft lip and palate. Aust Orthod J 2010;26:165-70.

27. Moreira I, Suri S, Ross B, Tompson B, Fisher D, et al. Soft-tissue profile growth in patients with repaired complete unilateral cleft lip and palate: a cephalometric comparison with normal controls at ages 7, 11, and 18 years. Am J Orthod Dentofacial Orthop 2014;145:341-58.

28. Lilja J. Alveolar bone grafting. Indian J Plast Surg 2009;42:S110-5.

29. Meazzini MC, Capasso E, Morabito A, Garattini G, Brusati R. Comparison of growth results in patients with unilateral cleft lip and palate after early secondary gingivoalveoloplasty and secondary bone grafting: 20 years follow up. Scand J Plast Reconstr Surg Hand Surg 2008;42:290-5. 